\title{
NON-LINEAR INTEGRAL EQUATIONS FOR HEUN FUNCTIONS
}

\author{
by B. D. SLEEMAN \\ (Received 14th August 1968)
}

\section{Introduction}

Some years ago Lambe and Ward (1) and Erdélyi (2) obtained integral equations for Heun polynomials and Heun functions. The integral equations discussed by these authors were of the form

$$
y(z)=\lambda \int_{C} t^{\gamma-1}(1-t)^{\delta-1}(1-t / a)^{\varepsilon-1} K(z, t) y(t) d t .
$$

Further, as is well known, the Heun equation includes, among its special cases, Lamés equation and Mathieu's equation and so (1.1) may be considered a generalisation of the integral equations satisfied by Lamé polynomials and Mathieu functions. However, integral equations of the type (1.1) are not the only ones satisfied by Lamé polynomials; Arscott (3) discussed a class of nonlinear integral equations associated with these functions. This paper then is concerned with discussing the existence of non-linear integral equations satisfied by solutions of Heun's equation.

In $\$ 2$ Heun's equation is given and Heun functions and Heun polynomials defined. $\$ 3$ is devoted to deriving the integral equation, while in $\$ 4$ the partial differential equation satisfied by the nucleus is solved. The method of solution is that of separation of the variables and gives rise to solutions in terms of products of hypergeometric functions. It is then demonstrated how suitable nuclei may be chosen in order to construct integral equations satisfied by Heun functions and Heun polynomials. Lamé's equation is considered as a special case.

\section{Definitions}

It is well known that, since any Fuchsian equation of the second order with four regular singularities preserves this character under any homographic transformation of the independent variable, three of the singularities can be brought to $z=0,1$ and infinity.

The fourth then becomes some finite point $z=a$ where, without loss of generality, we can specify $|a|>1$. Such Fuchsian equations may always be reduced to the standard equation

$$
\frac{d^{2} y}{d z^{2}}+\left\{\frac{\gamma}{z}+\frac{\delta}{z-1}+\frac{\varepsilon}{z-a}\right\} \frac{d y}{d z}+\frac{\alpha \beta(z-q) y}{z(z-1)(z-a)}=0,
$$


where

$$
1+\alpha+\beta-\gamma-\delta-\varepsilon=0,
$$

and $q$ is an arbitrary accessory parameter.

Equation (2.1) is called Heun's equation.

We adopt the following notation:

(i) The solution of the Heun equation which is analytic at the origin and normalised so that it takes the value unity there, will be denoted by

$$
H u(a, q ; \alpha, \beta, \gamma, \delta ; z)
$$

If no ambiguity is likely to arise, we may also adopt the more concise symbol,

$$
H u(q ; z) \text {. }
$$

The usual definition of a Heun function is given as that solution convergent in a region of the $z$-plane which includes at least two singularities of the Heun equation. In this paper, we shall be mainly concerned with Heun functions convergent in a region containing the singularities $z=0,1$, the analysis being readily adapted to deal with the other types of Heun function. Thus we define a Heun function as follows.

(ii) If, in addition to the properties given in (i), $H u(q ; z)$ is also regular at $z=1$, i.e., when $q$ is one of a set of characteristic values, Sleeman (4), then $H u(q ; z)$ becomes a Heun function and is represented by

$$
H u\left(q_{m} ; z\right), \quad m=1,2,3, \ldots
$$

(iii) If either $\alpha, \beta$ or both are negative integers ( $-n$, say) and $q$ takes characteristic values and the properties in (i) apply, then we obtain $n+1$ Heun polynomials, i.e. one for each value of $q$. Such functions are represented by

$$
H u\left(q_{m, n} ; z\right) \text {. }
$$

\section{The integral equation}

Theorem. Let (i) $w(z)$ be a solution of Heun's equation,

(ii) $H(z, s, t)$ be a solution of the partial differential equation

$$
(t-z) M_{s}(H)+(z-s) M_{t}(H)+(s-t) M_{z}(H)=0,
$$

where $M_{z}$ is a differential operator defined by

$$
\begin{aligned}
M_{z}(H) \equiv z(z-1)(z-a) \frac{\partial^{2} H}{\partial z^{2}} & \\
+ & {[\gamma(z-1)(z-a)+\delta z(z-a)+\varepsilon z(z-1)] \frac{\partial H}{\partial z}+\alpha \beta z H, }
\end{aligned}
$$

and $w(z), H(z, s, t)$ are analytic in appropriate complex regions,

(iii) $C_{1}, C_{2}$ be suitable paths in the complex $s$, t-planes such that both the quantities

$$
\left[s^{\gamma}(1-s)^{\delta}(1-s / a)^{\varepsilon}\left\{w(s) \frac{\partial H}{\partial s}-H \frac{\partial w(s)}{\partial s}\right\}\right]_{c_{1}}
$$


and

vanish,

$$
\left[t^{\gamma(1-t)^{\delta}(1-t / a)^{\varepsilon}}\left\{w(t) \frac{\partial H}{\partial t}-H \frac{\partial w(t)}{\partial t}\right\}\right]_{C_{2}}
$$

(iv) the function

$W(z)$

$=\int_{C_{1}} \int_{C_{2}}(s-t)(s t)^{y-1}\{(1-s)(1-t)\}^{\delta-1}\{(1-s / a)(1-t / a)\}^{e-1} H w(s) w(t) d s d t$

exist, and if the integral is singular, let it converge uniformly with respect to $z$ when $z, s, t$ lie in appropriate regions.

Then $W(z)$ is a solution of Heun's equation.

Proof. Consider the integral

$W(z)=\int_{C_{1}} \int_{C_{2}}(s-t)(s t)^{y-1}\{(1-s)(1-t)\}^{\delta-1}$

Then

$\{(1-s / a)(1-t / a)\}^{e-1} H w(s) w(t) d s d t$.

$$
\begin{aligned}
M_{z}(W) & =\int_{C_{1}} \int_{C_{2}}(s t)^{\gamma-1}\{(1-s)(1-t)\}^{\delta-1}\{(1-s / a)(1-t / a)\}^{z-1} w(s) w(t) \\
& =\int_{C_{1}} \int_{C_{2}}(s t)^{\gamma-1}\{(1-s)(1-t)\}^{\delta-1}\{(1-s / a)(1-t / a)\}^{z-1} w(s) w(t)
\end{aligned}
$$

using (3.1).

$$
\left\{(z-t) M_{s}(H)+(s-z) M_{t}(H)\right\} d s d t, \quad \text { (3.4) }
$$

Consider the integral

$\int_{C_{1}} \int_{C_{2}}(z-t)(s t)^{y-1}\{(1-s)(1-t)\}^{\delta-1}\{(1-s / a)(1-t / a)\}^{\varepsilon-1} w(s) w(t) M_{s}(H) d s d t$,

which on using the fact that

becomes

$$
s^{\gamma-1}(1-s)^{\delta-1}(1-s / a)^{\varepsilon-1} M_{s} \equiv \frac{\partial}{\partial s}\left\{a s^{\gamma}(1-s)^{\delta}(1-s / a)^{\varepsilon} \frac{\partial}{\partial s}\right\}
$$

$a \int_{C_{1}} \int_{C_{2}} t^{\gamma-1}(1-t)^{\delta-1}(1-t / a)^{\varepsilon-1} w(s) w(t)(z-t) \frac{\partial}{\partial s}\left\{s^{\gamma}(1-s)^{\delta}(1-s / a)^{\varepsilon} \frac{\partial H}{\partial s}\right\} d s d t$.

Integrating (3.5) with respect to $s$ by parts gives

$$
\begin{aligned}
\int_{C_{1}} a w(s) \frac{\partial}{\partial s}\left\{s^{\gamma}(1-s)^{\delta}(1-s / a)^{e}\right. & \left.\frac{\partial H}{\partial s}\right\} \\
& =\left[a s^{\gamma}(1-s)^{\delta}(1-s / a)^{2}\right]\left\{w(s) \frac{\partial H}{\partial s}-H \frac{\partial w(s)}{\partial s}\right\}_{C_{1}} \\
& +\int_{C_{1}} s^{\gamma-1}(1-s)^{\delta-1}(1-s / a)^{e-1} H M_{s}(w(s)) d s .
\end{aligned}
$$


If $C_{1}$ is chosen so that the integrated part vanishes, then (3.5) becomes

$\int_{C_{1}} \int_{C_{2}}(s t)^{\gamma-1}\{(1-s)(1-t)\}^{\delta-1}\{(1-s / a)(1-t / a)\}^{e-1}(z-t) H w(t) M_{s}(w(s)) d s d t$.

Similarly, if $C_{2}$ is such that

$$
\left[t^{\gamma}(1-t)(1-t / a)^{e}\left\{w(t) \frac{\partial H}{\partial t}-H \frac{\partial w(t)}{\partial t}\right\}\right]_{c_{2}}
$$

vanishes, then (3.4) can be rewritten as

$$
\begin{aligned}
M_{z}(W)=\int_{C_{1}} \int_{C_{2}}(s t)^{\gamma-1}\{(1-s) & (1-t)\}^{\delta-1}\{(1-s / a)(1-t / a)\}^{\varepsilon-1} H \\
& \times\left\{(z-t) w(t) M_{s}(w(s))+(s-z) w(s) M_{t}(w(t))\right\} d s d t .
\end{aligned}
$$

If we write Heun's equation as

$$
\left\{M_{z}-\alpha \beta q\right\} y=0
$$

then on substituting for $W(z)$ we have

$$
\begin{aligned}
\left\{M_{z}-\alpha \beta q\right\} W=\int_{C_{1}} \int_{C_{2}}(s t)^{\gamma-1}\{(1-s) & (1-t)\}^{\delta-1}\{(1-s / a)(1-t / a)\}^{\varepsilon-1} H \\
& \times\left[(z-t) w(t)\left\{M_{s}(w(s))-\alpha \beta q w(s)\right\}\right. \\
& \left.+(s-z) w(s)\left\{M_{t}(w(t))-\alpha \beta q w(t)\right\}\right] d s d t=0,
\end{aligned}
$$

since $w(s), w(t)$ are solutions of Heun's equation. Consequently, $W(z)$ is a solution of Heun's equation.

4. The solution of the partial differential equation satisfied by the nucleus

We introduce new variables $(u, v, w)$ defined by the relations

$$
\begin{gathered}
u=\frac{l}{a}(s t z)^{\frac{1}{2}}, \quad v=l\left\{\frac{(s-1)(t-1)(z-1)}{a(1-a)}\right\}^{\frac{1}{2}}, \\
w=i \frac{l}{a}\left\{\frac{(s-a)(t-a)(z-a)}{(1-a)}\right\}^{\frac{1}{2}},
\end{gathered}
$$

in which $l$ is an arbitrary parameter.

In terms of these new variables, equation (3.1) becomes, after tedious but straightforward algebra,

where

$$
\nabla^{2} H+(2 \gamma-1)\left(\frac{1}{u} \frac{\partial H}{\partial u}+\frac{1}{v} \frac{\partial H}{\partial v}+\frac{1}{w} \frac{\partial H}{\partial w}\right)=0
$$

$$
\nabla^{2}=\frac{\partial^{2}}{\partial u^{2}}+\frac{\partial^{2}}{\partial v^{2}}+\frac{\partial^{2}}{\partial w^{2}}
$$

In the special case $\gamma=\frac{1}{2}$ equation (4.2) reduces to Laplace's equation so that it is easy to write down suitable nuclei. 
We now make the further transformation to the variables $(r, \theta, \phi)$ related to $(u, v, w)$ by

$$
u=r \cos \theta, \quad v=r \sin \theta \sin \phi, \quad w=r \sin \theta \cos \phi .
$$

Under this transformation (4.2) becomes

$$
\begin{aligned}
\frac{\partial^{2} H}{\partial r^{2}}+\frac{6 \gamma-1}{r} \frac{\partial H}{\partial r}+\frac{1}{r^{2}}\left[\frac{\partial^{2} H}{\partial \theta^{2}}\right. & \left.+\{(4 \gamma-1) \cot \theta-(2 \gamma-1) \tan \theta\} \frac{\partial H}{\partial \theta}\right] \\
+ & \frac{1}{r^{2} \sin ^{2} \theta}\left[\frac{\partial^{2} H}{\partial \phi^{2}}+2(2 \gamma-1) \cot 2 \phi \frac{\partial H}{\partial \phi}\right]=0 .
\end{aligned}
$$

Consequently, in the case $\gamma=\frac{1}{2}$ we obtain suitable nuclei for (3.3) in terms of spherical harmonics in $(r, \theta, \phi)$.

A separated solution of (4.4)

$$
H=R(r) G(\theta) K(\phi),
$$

is obtained if $R, G$ and $K$ satisfy the ordinary differential equations

and

$$
\begin{gathered}
r^{2} \frac{d^{2} R}{d r^{2}}+(6 \gamma-1) r \frac{d R}{d r}-\lambda R=0, \\
\sin ^{2} \theta\left[\frac{d^{2} G}{d \theta^{2}}+\{(4 \gamma-1) \cot \theta-(2 \gamma-1) \tan \theta\} \frac{d G}{d \theta}+\lambda G\right]-\mu G=0
\end{gathered}
$$

$$
\frac{d^{2} K}{d \phi^{2}}+2(2 \gamma-1) \cot 2 \phi \frac{d K}{d \phi}+\mu K=0
$$

$\lambda, \mu$ being separation constants.

A fundamental pair of solutions of (4.6) are

$$
r^{m_{1}}, r^{m_{2}}
$$

where $m_{1}, m_{2}$ are the roots, assumed distinct, of the equation

$$
m^{2}+2(3 \gamma-1) m-\lambda=0 \text {. }
$$

If we put $\xi=\cos ^{2} \theta$ then we find that (4.7) takes the form

$$
\xi(1-\xi) \frac{d^{2} G}{d \xi^{2}}+(\gamma-3 \gamma \xi) \frac{d G}{d \xi}+\left(\lambda / 4-\frac{\mu / 4}{1-\xi}\right) G=0
$$

If $p$ satisfies the equation then

$$
p^{2}+(2 \gamma-1) p-\mu / 4=0
$$

$$
W(\xi)=(1-\xi)^{-p} G(\xi)
$$

satisfies the hypergeometric equation

$$
\xi(1-\xi) \frac{d^{2} W}{d \xi^{2}}+\{\gamma-(3 \gamma+2 p) \xi\} \frac{d W}{d \xi}+\left(\lambda / 4+p-3 \gamma p-p^{2}\right) W=0 .
$$


Thus (4.7) has the solutions

where

$$
\sin ^{2 p} \theta P\left\{\begin{array}{cccc}
0 & 1 & \infty & \\
0 & 0 & a & \cos ^{2} \theta \\
1-\gamma & 1-2 p-2 \gamma & b
\end{array}\right\}
$$

$$
a, b=\frac{(3 \gamma+2 p-1) \pm\left\{(3 \gamma-1)^{2}+\lambda\right\}^{\frac{1}{2}}}{2}
$$

and $p$ is a root of (4.11). Similarly, (4.8) has the solutions

where

$$
P\left\{\begin{array}{cccc}
0 & 1 & \infty & \\
0 & 0 & a^{\prime} & \cos ^{2} \phi \\
1-\gamma & 1+\gamma & b^{\prime} &
\end{array}\right\}
$$

$$
a^{\prime}, b^{\prime}=\frac{(2 \gamma-1) \pm\left\{(2 \gamma-1)^{2}+\mu\right\}^{\frac{1}{2}}}{2} .
$$

The variables $(r, \theta, \phi)$ are given by

$$
\begin{aligned}
r^{2} & =\frac{l^{2}}{a}(s+t+z-1-a), \\
\cos ^{2} \theta & =\frac{s t z}{a(s+t+z-1-a)} \\
\cos ^{2} \phi & =\frac{(s-a)(t-a)(z-a)}{(1-a)(s t z-a)(s+t+z-1-a)} .
\end{aligned}
$$

Thus typical solutions of the equation for the nucleus are

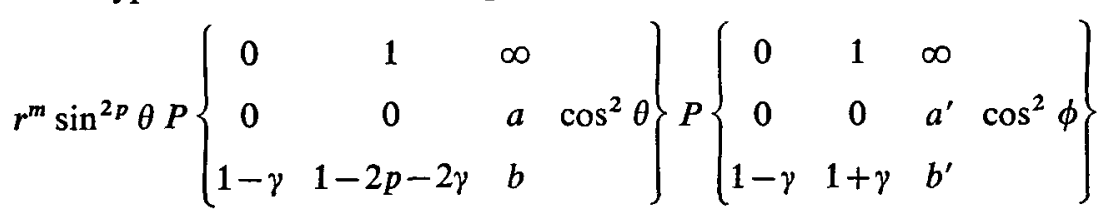

The expression (4.19) gives a very wide range of nuclei suitable for integral equations or relations for solutions of the Heun equation. The nuclei can be chosen as combinations of the various branches of the $P$-functions involved and may even be linear combinations summed over the separation constants $\lambda$ and $\mu$. We shall demonstrate the use of (4.19) in constructing suitable nuclei for integral equations satisfied by Heun functions and Heun polynomials. We shall take $C_{1}$ to be a contour enclosing the points $s=0,1$ and $C_{2}$ as a similar contour enclosing the points $t=0,1$. In the case of the Heun function regular at the singularities $z=0,1$, we choose that nucleus which, when considered as a function of $z$, is also regular at $z=0,1$. This can be achieved by taking that 
branch of the first $P$-function in (4.19) which, as a function of $z$, is regular at the origin and belongs to the exponent 0 there and that branch of the second $P$-function which, as a function of $z$ is regular at $z=1$ and belongs to the exponent 0 there. Such a nucleus is of the form

$$
r^{m} \sin ^{2 p} \theta_{2} F_{1}\left(a, b ; \gamma ; \cos ^{2} \theta\right){ }_{2} F_{1}\left(a^{\prime}, b^{\prime} ; \gamma ; \sin ^{2} \phi\right) .
$$

The contours $C_{1}, C_{2}$ must be chosen so that the singularities where $\cos ^{2} \theta=1$ or $\infty$ are excluded, i.e. where

$$
a(s+t+z-1-a)=s t z \text { or } 0 .
$$

Then, with the nucleus (4.20), the " integrated parts" $(3.2 a, b)$ vanish if $C_{1}, C_{2}$ are each taken to be Pochhammer loop contours about the points 0 and 1 . The integral (3.3) thus represents a constant multiple (possibly zero) of the Heun function. If, however, $\operatorname{Re} \gamma>0$ or $\operatorname{Re} \delta>0$, and the separation constants $\lambda$ and $\mu$ are chosen to make $\operatorname{Re}(\gamma-a-b)>0$ and $\operatorname{Re}\left(\gamma-a^{\prime}-b^{\prime}\right)>0$, so that the hypergeometric functions in (4.20) are regular at $\cos ^{2} \theta=\sin ^{2} \phi=1$, then the contours of integration may be deformed into simple loop contours or straight lines.

In the case of Heun polynomials, the nucleus, when considered as a function of $z$, must be finite for finite values of $z$ and also regular at the origin. Further, if $H u(q, z)$ is a polynomial of degree $n$ in $z$, then the nucleus must also be a polynomial of degree $n$ in $z$. If we again consider the nucleus (4.20), then at least one of $a, b$ and at least one of $a^{\prime}, b^{\prime}$ must be negative integers.

If

then

$$
\lambda=4(3 \gamma-1) n+4 n^{2}, \quad n=0,1,2, \ldots,
$$

If

$$
a=(3 \gamma-1)+p+n \text { and } b=p-n \text {. }
$$$$
\mu=4(2 \gamma-1) s+4 s^{2}, \quad s=0,1,2, \ldots,
$$

then

$$
a^{\prime}=(2 \gamma-1)+s, \quad b^{\prime}=-s,
$$

and $m=2 n$ or $-2(3 \gamma-1)-2 n$ and $p=s$ or $-(2 \gamma-1)-s$. Hence

or

$$
a=(3 \gamma-1)+s+n, \quad b=s-n
$$

By taking

and

$$
a=\gamma-s+n, \quad b=-(2 \gamma-1)-s-n .
$$

$$
m=2 n, \quad a=(3 \gamma-1)+s+n, \quad b=s-n
$$

$$
a^{\prime}=(2 \gamma-1)+s, \quad b^{\prime}=-s,
$$

then, if $s-n$ and $-s$ are negative integers, the nucleus, considered as a function of $z$ is finite for finite values of $z$ and is regular at $z=0$. It is therefore a suitable nucleus for the Heun polynomial of degree $n$ in $z$ if $s$ takes one of the values $s=0,1, \ldots, n$. In fact, we could choose a nucleus which is the sum (over $s$ ) of nuclei of the form (4.20). Thus we see that, for one type of Heun polynomial of degree $n$, there are $(n+1)$ possible nuclei of the form (4.20). If $\operatorname{Re} \gamma>0$, 
$\operatorname{Re} \delta>0$ then the contours $C_{1}, C_{2}$ may be replaced by the straight line intervals $s=(0,1)$ and $t=(0,1)$ respectively. Obviously, since the hypergeometric functions in (4.20) are now polynomials, the further restrictions $\operatorname{Re}(y-a-b)>0$ and $\operatorname{Re}\left(\gamma-a^{\prime}-b^{\prime}\right)>0$ do not apply. In the above analysis we have taken $C_{1}, C_{2}$ to be contours surrounding the points $s=(0,1)$ and $t=(0,1)$ or with certain restrictions on the parameters, straight lines joining them. If, in the case of Heun functions, the restrictions $\operatorname{Re} \gamma>0, \operatorname{Re} \delta>0, \operatorname{Re} \varepsilon>0$ are applied, then $C_{1}, C_{2}$ may be taken to be any pair of the straight lines joining the points $(0,1)$, $(0, a),(1, a)$ in the complex $s, t$-planes. When $C_{1}, C_{2}$ are the same then only two of the above restrictions are necessary. For Heun polynomials the same arguments apply, except that here the restrictions may be removed if $C_{1}, C_{2}$ are Pochhammer loop contours surrounding any of the above pairs of points.

An important special case which may be considered is that in which the parameters in Heun's equation take the special values $\alpha=-\frac{1}{2} n, \beta=\frac{1}{2}(n+1)$ ( $n$ being a non-negative integer) $\gamma=\frac{1}{2}=\delta=\varepsilon$ and $a=k^{-2}$ where $k$ is the modulus of the Jacobian elliptic function snz. Heun's equation now takes the algebraic form of Lamé's equation,

$$
\frac{d^{2} y}{d z^{2}}+\frac{1}{2}\left\{\frac{1}{z}+\frac{1}{z-1}+\frac{1}{z-k^{-2}}\right\} \frac{d y}{d z}+\frac{\left(h k^{-2}-n(n+1) z\right) y}{4 z(z-1)\left(z-k^{-2}\right)}=0 .
$$

Here $h k^{-2}=n(n+1) q$.

If we put $z=\operatorname{sn}^{2} u$ we obtain the Jacobian form of Lamé's equation namely,

$$
\frac{d^{2} y}{d u^{2}}+\left\{h-n(n+1) k^{2} \operatorname{sn}^{2} u\right\} y=0
$$

Further, if $h$ is a root of a certain characteristic equation, then Lamé polynomials $E_{n}^{m}(u)$ are obtained, where $m=0,1,2, \ldots, N$ and $N=\left[\frac{1}{2} n\right]$. The notation adopted here and in the subsequent discussion is due to Arscott (5).

With reference to the nucleus (4.20) we find, for

$$
\gamma=\frac{1}{2}, \quad m=2 n, \quad p=s, \quad a=s+n+\frac{1}{2}, \quad b=s-n, \quad a^{\prime}=s, \quad b^{\prime}=-s .
$$

and thus (4.20) becomes a constant multiple, dependent on $n$ and $s$, of the spherical harmonic

If in (4.18) we put

$$
r^{2 n} P_{2 n}^{2 s}(\cos \theta) \cos 2 s \phi .
$$

$z=\operatorname{sn}^{2} \alpha, s=\operatorname{sn}^{2} \beta, \quad t=\operatorname{sn}^{2} \gamma, \quad a=k^{-2}$

$$
\begin{gathered}
r^{2}=l^{2}\left(k^{2} \operatorname{sn}^{2} \alpha-k^{2} \operatorname{cn}^{2} \beta-\operatorname{dn}^{2} \gamma\right) \\
\cos ^{2} \theta=\frac{k^{2} \operatorname{sn}^{2} \alpha \operatorname{sn}^{2} \beta \operatorname{sn}^{2} \gamma}{\left(k^{2} \operatorname{sn}^{2} \alpha-k^{2} \operatorname{cn}^{2} \beta-\operatorname{dn}^{2} \gamma\right)}
\end{gathered}
$$

and

$$
\cos ^{2} \phi=\frac{\operatorname{dn}^{2} \alpha \mathrm{dn}^{2} \beta \mathrm{dn}^{2} \gamma}{k^{\prime 2}\left[k^{4} \operatorname{sn}^{2} \alpha \operatorname{sn}^{2} \beta \operatorname{sn}^{2} \gamma-\left(k^{2} \operatorname{sn}^{2} \alpha-k^{2} \operatorname{cn}^{2} \beta-\mathrm{dn}^{2} \gamma\right)\right]}
$$


Obviously we may take $C_{1}, C_{2}$ to be the straight lines joining $s=(0,1)$, $t=(0, a)$ and in the transformed plane these are taken to be the segments $(-2 K, 2 K)$ for $\beta$ and $\left(K-2 i K^{\prime}, K+2 i K^{\prime}\right)$ for $\gamma$. From these results we see that the nucleus (4.30) is suitable for integral equations satisfied by the Lamé polynomial $u E_{2 n}^{m}(\alpha)$. That is, we have the result

$$
u E_{2 n}^{m}(\alpha)=\lambda \int_{-2 K}^{2 K} \int_{K-2 i K^{\prime}}^{K+2 i K^{\prime}} r^{2 n} P_{2 n}^{2 s}(\cos \theta) \cos 2 s \phi\left(\operatorname{sn}^{2} \beta-\operatorname{sn}^{2} \gamma\right) u E p_{2 n}^{m}(\beta, \gamma) d \beta d \gamma,
$$

for any $s \leqq n$.

It is important to point out that, in the case of Lamé's equation, the equation satisfied by the nucleus is Laplace's equation and, consequently, it is a simple matter to write down suitable nuclei without appealing to the form (4.19).

Integral equations of the type (4.35) have been discussed by Arscott (3) and Sleeman (6). Finally we remark that, although the nuclei (4.19) may, as discussed above, be used to construct integral equations satisfied by Heun functions and Heun polynomials, they may also be used to construct integral representations between the various solutions of Heun's equation. The number of possible cases to be considered here is extremely large and will be treated in a subsequent paper.

The author is indebted to Professor F. M. Arscott for his encouragement during the progress of this work.

\section{REFERENCES}

(1) C. G. LAmbe and D. R. WARD, Some differential equations and associated integral equations, Quart. J. Math. Oxford ser., 5 (1934), 81-97.

(2) A. ERDÉLYI, Integral equations for Heun functions, Quart. J. Math. Oxford ser., 13 (1942), 107-112.

(3) F. M. ARscotr, Integral equations and relations for Lamé functions, Quart. J. Math. Oxford ser. (2), 15 (1964), 103-115.

(4) B. D. SLeEmAN, Integral representations for solutions of Heun's equation, Proc. Camb. Phil. Soc., 65 (1969), 447-459. 1964).

(5) F. M. Arscott, Periodic Differential Equations (Pergamon Press, Oxford,

(6) B. D. SLeeman, Integral equations and relations for Lamé functions and ellipsoidal wave functions, Proc. Camb. Phil. Soc. 64 (1968), 113-126.

DepartMent OF Mathematics,

THE UNIVERSITY, DUNDEE

E.M.S.-S 